\title{
Performance of a TV white space database with different terrain resolutions and propagation models
}

\author{
A. M. Fanan, N. G. Riley, M. Mehdawi, and O. Alfahad
}

\begin{abstract}
Cognitive Radio has now become a realistic option for the solution of the spectrum scarcity problem in wireless communication. TV channels (the primary user) can be protected from secondary-user interference by accurate prediction of TV White Spaces (TVWS) by using appropriate propagation modelling. In this paper we address two related aspects of channel occupancy prediction for cognitive radio. Firstly we investigate the best combination of empirical propagation model and spatial resolution of terrain data for predicting TVWS by examining the performance of three propagation models (Extended-Hata, Davidson-Hata and Egli) in the TV band 470 to $790 \mathrm{MHz}$ along with terrain data resolutions of 1000,100 and $30 \mathrm{~m}$, when compared with a comprehensive set of propagation measurements taken in randomly-selected locations around Hull, UK. Secondly we describe how such models can be integrated into a databasedriven tool for cognitive radio channel selection within the TVWS environment.
\end{abstract}

Keywords - Path loss, Diffraction, Propagation Model, Spectrum Measurement, TVWS, Cognitive Radio.

\section{INTRODUCTION}

A $\mathrm{S}$ radios in future wireless systems become more flexible and reconfigurable and available radio spectrum becomes scarce, there is the possibility of using TV white space devices (WSDs) as secondary users in the Broadcast bands without causing harmful interference to licensed incumbents. Currently, one candidate method could be to utilise a geo-location database approach. The white space device should be able to determine available channel opportunities for a given location by accessing a database of TV white spaces channels including data on each transmitter and each site, variable channels, transmitter power, and time of validation [1]. Therefore, the TV channel (primary user) can be protected from harmful

Paper received April 24, 2017; revised October 31, 2017; accepted November 5, 2017. Date of publication December 25, 2017. The associate editor coordinating the review of this manuscript and approving it for publication was Prof. Aleksandar Nešković.

This paper is a revised and expanded version of the paper presented at the 24th Telecommunications Forum TELFOR 2016 [10].

A. M. Fanan, School of Engineering, University of Hull, Cottingham Road, Hull, HU6 7RX, UK (e-mail: anuarfanan@yahoo.com).

N. G. Riley, School of Engineering, University of Hull, Cottingham Road, Hull, HU6 7RX, UK (e-mail: n.g.riley@hull.ac.uk).

M. Mehdawi, School of Engineering, University of Hull Cottingham Road, Hull, HU6 7RX, UK (e-mail: m.a.mehdawi@2010.hull.ac.uk).

O.Alfahad, School of Engineering, University of Hull, Cottingham Road, Hull, HU6 7RX, UK (e-mail: o.a.alfahad@2014.hull.ac.uk). interference by accurately predicting TV White Spaces using an appropriate propagation model. Accurate prediction of radio propagation is considered essential in any wireless network, influencing deployment and management strategies with a suitable design for each network. In this paper we compare empirical propagation models for predicting TV white spaces by examining the applicability of three propagation models, Extended Hata, Davidson and Egli when used in the TV band from 470 to $790 \mathrm{MHz}$ based on real terrain data for the specific region of Yorkshire, UK. A comprehensive set of propagation measurements has been conducted in many random locations around Hull, UK.

Agreement between the measured and predicted values of path loss has been investigated, using MATLAB to analyse and compare the variation of path loss between the measured and predicted values.

The terrain profile was calculated by using the terrain database Globall and then taken into account in selected propagation models. The flexible cognitive TVWS database system was built using different propagation models to calculate available channels in each pixel of the selected area.

\section{Digital Terrain Elevation Data (DTED)}

DTED was developed by the US Defence Mapping Agency (DMA) and can be used to improve signal detection accuracy. Currently, DTED has six levels of spatial resolution, some of which are available to the public whilst others are only for military use [2].

Table 1: Digital Terrain Elevation

DATA RESOLUTION LEVELS.

\begin{tabular}{|l|l|l|l|l|c|}
\hline $\begin{array}{l}\text { DTED } \\
\text { Level }\end{array}$ & $\begin{array}{l}\text { Post } \\
\text { Spacing }\end{array}$ & $\begin{array}{l}\text { Ground } \\
\text { Dist }\end{array}$ & $\begin{array}{l}\text { Row } \mathrm{x} \\
\text { Column }\end{array}$ & Tile size & Av \\
\hline 0 & $30 \mathrm{~s}$ & $\sim 1 \mathrm{~km}$ & $121 \times 121$ & $1 \times 1$ degree & $\mathrm{Y}$ \\
\hline 1 & $3.0 \mathrm{~s}$ & $\begin{array}{l}\sim \\
100 \mathrm{~m}\end{array}$ & $\begin{array}{l}1200 \times \\
1200\end{array}$ & $1 \times 1$ degree & $\mathrm{Y}$ \\
\hline 2 & $1.0 \mathrm{~s}$ & $\sim 30 \mathrm{~m}$ & $\begin{array}{l}3600 \times \\
3600\end{array}$ & $1 \times 1$ degree & $\mathrm{Y}$ \\
\hline 3 & $0.333 \mathrm{~s}$ & $\sim 10 \mathrm{~m}$ & $900 \times 900$ & $5 \times 5$ minute & $\mathrm{N}$ \\
\hline 4 & $0.111 \mathrm{~s}$ & $\sim 3 \mathrm{~m}$ & $540 \times 540$ & $1 \times 1$ minute & $\mathrm{N}$ \\
\hline 5 & $\begin{array}{l}0.0370 \\
\mathrm{~s}\end{array}$ & $\sim 1 \mathrm{~m}$ & $810 \times 810$ & $30 \times 30 \mathrm{sec}$ & $\mathrm{N}$ \\
\hline
\end{tabular}

The elevation data of the resolution from 30 Arc to 1 Arc (level 0,1 and 2) are avaliable for public use and can be downloaded as different DTED extension files in each resolution. The USGS web application has been used to define the desired research region by specifying latitude and 
longitude as shown in Fig. 1, which identifies the required terrain tiles. Fig. 2 classifies all tiles, identified by the web application earthexplorer.usgs.gov.

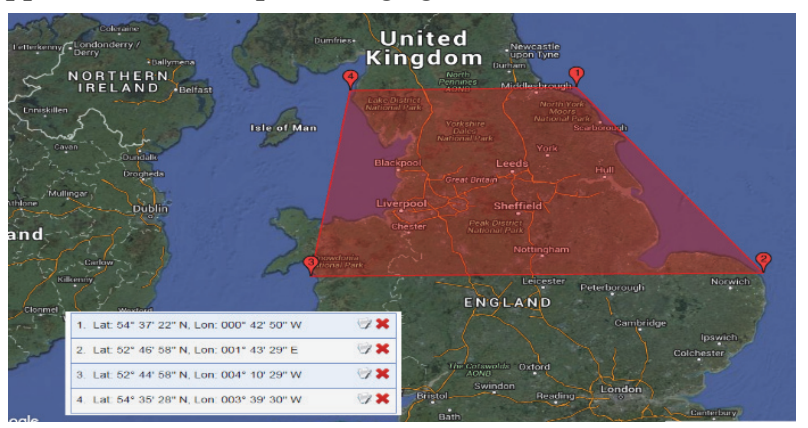

Fig. 1. Region selected of terrain data.

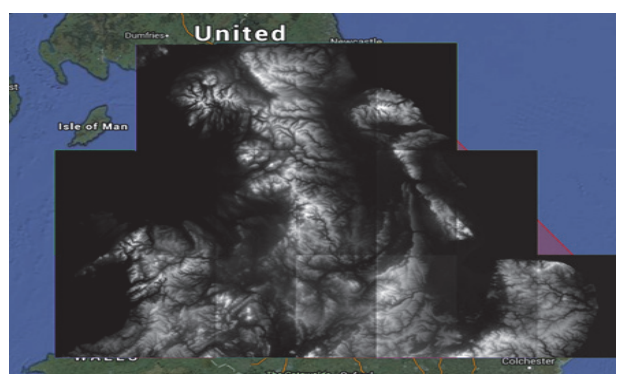

Fig. 2. Research region of terrain elevation data by NASA's shuttle radar topography mission (SRTM) with resolution 1 Arc second $(30 \mathrm{~m})$.

\section{TVWS GEOLOCATION DATABASE}

The use of a TV white space geolocation database enables the most effective detection method to predict available channels and calculate TV coverage maps for each pixel in the selected region by using an appropriate propagation model, selected for accuracy and efficiency. This kind of technique can avoid signal detection problems caused by shadow fading effects. Construction of the geolocation database requires primary user information including frequency of operation, transmitted power, location, transmission time and height and type of transmit antenna. This information will protect spectrum incumbents from interference from secondary users who will access the database by sending a query to obtain available channels in a given area at a certain time. Furthermore, the geolocation database might have proxy to make queries and identify available channels for WSDs [3].

\section{Propagation Models}

When planning wireless communication systems and designing wireless networks, the accuracy of the prediction of propagation characteristics of each environment should be taken into account. One of the most significant parameters, which can be provided by propagation prediction, is large-scale path loss, which affects directly the coverage of a base station placement and its performance. However, using field measurements to obtain these parameters without depending on propagation models is time-consuming and costly. The following subsections provide a brief explanation of several empirical propagation models [4]. The models discussed are the Extended Hata, Davidson-Hata and Egli models.

\section{A. Extended Hata Model}

The Extended Hata model was derived from HataOkumura which has some factors that depend on the type of environment. This model has added other correction factors to meet the requirements of IRU-R and for extending range up to $100 \mathrm{~km}$ as shown in the following equation.

$$
\begin{aligned}
& L(\mathrm{~dB})=69.55+26.16 \log _{10}\left(f_{M H z}\right)-13.82 \log _{10}\left(h_{t}\right) \\
& -a\left(h_{r}\right)+\left(44.9-6.55 \log _{10} h_{t}\right)\left(\log _{10}\left(d_{k m}\right)\right)^{b}-K
\end{aligned}
$$

where $f$ represents the carrier frequency (150 to $1500 \mathrm{MHz}$ ), $h_{t}$ is the height of the base station antenna (m) and $h_{r}$ is the height of receive antenna $(\mathrm{m})$. The distance from transmitter to receiver is $d \mathrm{~km}$. The correction factors $a\left(h_{r}\right)$ and $K$ depend on the type of environment whereas the factor $b$ also depends on the path length [5].

\section{B. Davidson-Hata Model}

This model is based on modification of several corrections to Hata's formulas to extend the maximum distance to $300 \mathrm{~km}$ and frequency range from 30 to $1500 \mathrm{MHz}$ as mentioned in the publication TSB-88A by the Telecommunications Industry Association (TIA). The path loss of the Davidson model is illustrated in the following.

$$
\begin{aligned}
& P L_{\text {David }}=P L_{\text {Hata }}+A\left(h_{t}, d_{k m}\right)-S_{1}\left(d_{k m}\right)-S_{2}\left(h_{t}, d_{k m}\right) \\
& -S_{3}\left(f_{M H z}\right)-S_{4}\left(f_{M H z}, d_{k m}\right) . \\
& P L_{\text {Hata }}=69.55+26.16 \log _{10}\left(f_{M H z}\right)-13.82 \log _{10}\left(h_{t}\right) \\
& -a\left(h_{r}\right)+\left(44.9-6.55 \log _{10} h_{t}\right) \log _{10}\left(d_{k m}\right)
\end{aligned}
$$

The factors which extend distance up to $300 \mathrm{~km}$ are represented as $A$ and $S_{1} . S_{2}$ is a correction factor to extend the height of base station antenna to $2500 \mathrm{~m}$, while the factors $S_{3}$ and $S_{4}$ extend the frequency range over 30 to $1500 \mathrm{MHz}$ [6]. " $a\left(h_{r}\right)$ ” is a correction factor for receiver antenna height.

\section{Egli Model}

This model is commonly used for point to point communication to predict path loss in an urban or rural area, where transmission has to go over an irregular terrain between a fixed transmitter and receiver in the frequency range 40 to $900 \mathrm{MHz}$. The path loss equation for the Egli model can be written as follows:

$$
\begin{aligned}
& P L_{E g l i}= \begin{cases}20 \log _{10}\left(f_{M H z}\right)+p_{0}+76.3, & h_{2}<10 \\
20 \log _{10}\left(f_{M H z}\right)+p_{0}+83.9, & h_{2}<10\end{cases} \\
& p_{0}=40 \log _{10}\left(d_{k m}\right)-20 \log _{10}\left(h_{t}\right)-10 \log _{10}\left(h_{r}\right)
\end{aligned}
$$

where $h_{t}$ is the height of transmitter antenna $(\mathrm{m}), h_{r}$ is the height of receiver antenna $(\mathrm{m})$, the distance between transmitter and receiver is denoted by $d \mathrm{~km}$ and the transmission frequency is represented as $f(\mathrm{MHz})$ [7].

\section{Field Measurement and Data Collection}

The main goal of selection different locations for conducting various measurements is to examine the signal strength behavior in different environments at various distances from the transmitter and to observe how the terrain affects the received signal. The measurements have been taken at 23 locations distributed randomly around Kingston-upon Hull, UK as shown in Fig. 3. 


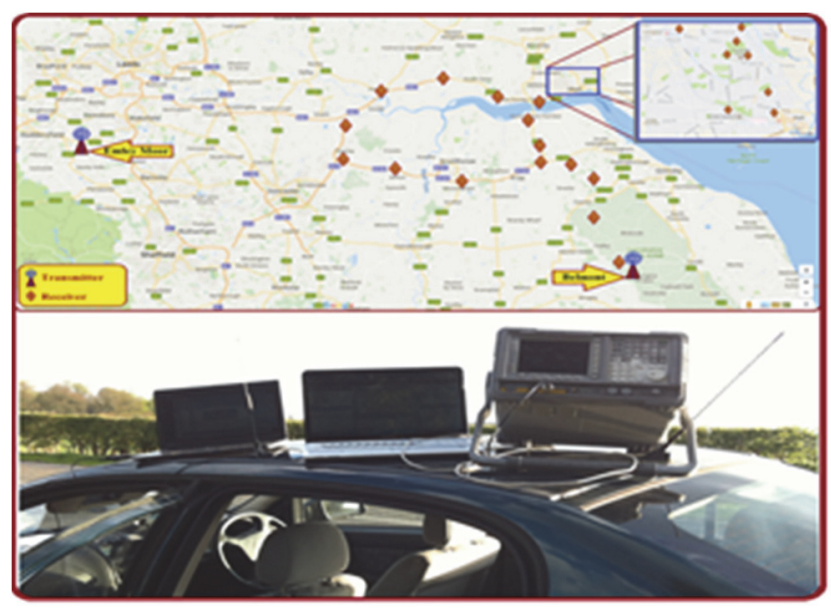

Fig. 3. Measurement sites and geographical location in Hull and surrounding areas.

The main equipment employed in this includes an omnidirectional antenna (covering the frequency range 174 to $230 \mathrm{MHz}$ (VHF) and 470 to $790 \mathrm{MHz}$ (UHF) with gain of $3.5 \mathrm{dBi}$ ) and spectrum analyser (Agilent E4407B, frequency range $9 \mathrm{kHz}$ to $26.5 \mathrm{GHz}$ ) which was connected with a laptop computer by using a general purpose interface bus. A Matlab program on the laptop received raw data and stored them in binary files. In addtion, the measurement locations were determined by using a mobile GPS application.

\section{ANAlysis Of Models’ Performance}

\section{A. Propagation Path Loss Analysis}

The main criterion for model assessment is path loss. A simulation program was implemented in Matlab, using channel 33 to conduct the comparison between the three propagation models and the measured results. In order to compare the real measurements with different propagation models, the path loss should be extracted from the real measurements by using the following equation in each location [8].

$$
P L=T X+T X_{\text {Gain }}+P R_{\text {Gain }}-R P
$$

where $T X$ denotes the transmitted power, transmitting antenna gain is represented as $T X_{\text {Gain }}, P L$ is the path loss, receiving antenna gain is denoted as $P R_{\text {Gain }}$ and $R P$ is the received power, $\mathrm{dBm}$.

To evaluate the propagation models against real measurements, several equations might be used to identify the most accurate propagation model. The error between predicted and measured path loss values was calculated by equation (8) and mean square error (MSE) calculated by equation (9).

$$
\begin{gathered}
E_{i}=M_{i}-P r_{i} \\
M S E=\frac{\sum_{i=1}^{n}\left(E_{i}\right)^{2}}{N}
\end{gathered}
$$

in which $E_{i}$ denotes the difference between the predicted model path losses $P r_{i}$ and real measured path loss $M_{i}$ derived from measured received power in each location. Equations 8 and 9 are then used to calculate the standard deviation (SD), equation (10), whilst root mean square error (RMSE) is calculated by equation (11), which also depends on MSE as calculated in equation (9).

$$
\begin{aligned}
S D & =\sqrt{\frac{\sum_{i=1}^{n}\left(E_{i}\right)^{2}}{N-1}} \\
R M S E & =\sqrt{\frac{1}{N} \sum_{i=1}^{n}\left(E_{i}\right)^{2}}
\end{aligned}
$$

\section{B. Terrain Profile-based Diffraction model}

One of the main effects of the terrain profile is to cause diffraction or bending of EM waves around obstacles such as mountains, hills or man-made structures which obscure the direct path. Diffraction is a phenomenon resulting from the wave property of light and radiowaves. The most common model of diffraction is single knife edge diffraction, explained by Huygens's Principle [9].

In previous work [10], we considered terrain resolutions of 1 and 30 Arc seconds, and determined that each of them had advantages and disadvantages in terms of accuracy and calculation time.

In this work, we attempt to improve these results by investigating a third resolution value between 1 and 30 Arc seconds to improve the compromise between accuracy and implementation time. Whilst calculating diffraction using the three different resolutions and investigating its effect on the received signal, we noticed that in the location approximately $38 \mathrm{~km}$ along the path shown in Fig. 4, in the 30 Arc second resolution the elevation value is $100 \mathrm{~m}$, whilst when using 3 Arc second and 1 Arc second resolution, the elevation values are approximately 86 and $83 \mathrm{~m}$ respectively.

\section{COMPARISON AND RESUlTS}

The measurement study covered the area around the city of Hull, which was represented to measure the UHF TV band from 470 to $790 \mathrm{MHz}$ with consideration of all radio and TV stations that feed the whole Hull area. Most of the channels transmitted into the area originate from the Belmont and Emley Moor transmitters. The results of comparison of predicted path loss with measurements for two cases (excluding and including terrain modelling) are presented by using the previously defined criteria MSE, SD and RMSE. Fig. 5 shows an example of the results including path loss curves for each propagation model and a table of calculated parameters. This example is for the case with no terrain model.

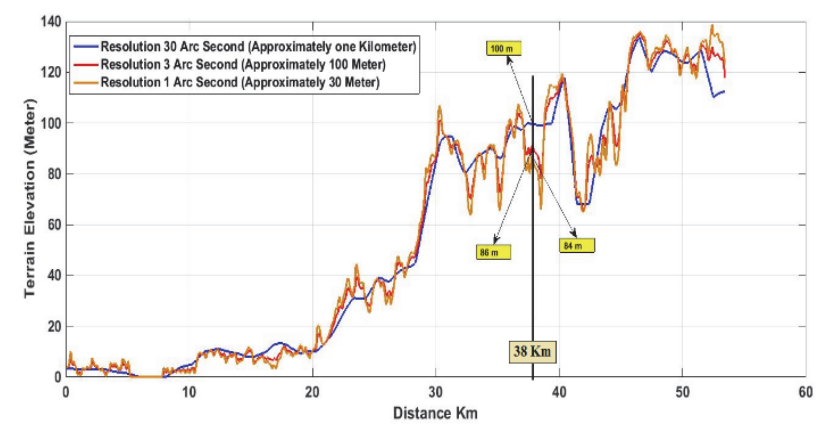

Fig. 4. Terrain elevation data of the path from university of Hull to Belmont TV Transmitter in different resolutions. 
This analysis may be undertaken for all selected measurement points, by flexible selection of the transmitter name, terrain resolution and transmitted channel. Results corresponding to all measurement locations and comparison of the three propagation models with real measurements along with parameter analysis, are dicussed and classified in the following sections.

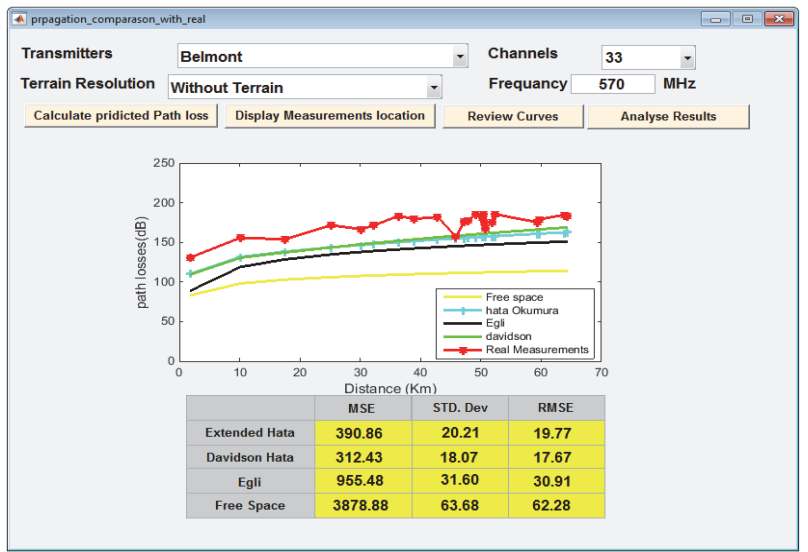

Fig. 5. Example of propagation model comparison in the selected measurement locations.

\section{A. Comparison of Propagation Models without Terrain}

The propagation modelling, excluding diffraction path loss factor are compared with measured data as shown in Fig. 6. It is seen that the Davidson model provides the best comparison with measured data. The calculated parameters for this case are presented in Table 2. The Extended Hata model compares well with the Davidson model for distances less than approximately $35 \mathrm{~km}$, but at larger ranges path loss increases slightly less than in the value Davidson model. The Egli model produces results consistently 8-10 dB less than the Extended Hata results. The difference of the path loss between measurement and proposed models was increasing slightly until approximately $47 \mathrm{~km}$ with a difference ranging between 30 and $40 \mathrm{~dB}$. After this distance, the difference was very variable between about 0 and $30 \mathrm{~dB}$. It is therefore suggested that several factors should be included in the propagation modelling such as diffraction, Earth radius, reflection and clutter each of which can impact significantly the behavior of the propagation models.

Table 2 shows the error statistics for each propagation model results by using the evaluation criteria mentioned above. Davidson Hata gives the best result among other models with RMSE of $17.67 \mathrm{~dB}$, which is however not considered as a good result, while the Extended Hata model gives slightly worse results with RMSE $19.77 \mathrm{~dB}$. The main reason is likely to be that their equations were derived from the Hata Okumura model.

The worst performance was represented by the Egli and free space models with high values of RMSE. In summary the lowest value of RMSE is $17.67 \mathrm{~dB}$, which is nevertheless not considered as a good enough result to be the basis of spectrum occupancy decision making. Thus, to improve RMSE results, it is necessary to include a terrain model when calculating the path loss in the propagation models.

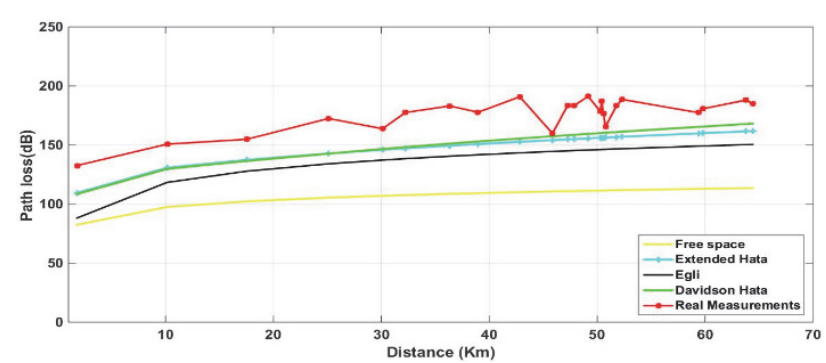

Fig. 6. Propagation models without terrain.

Table 2: Fitted Propagation Models Without TERRAIN AT HULL-UK.

\begin{tabular}{|l|c|c|c|}
\hline Model & MSE & STD. Dev & RMSE \\
\hline Extended Hata & 390.86 & 20.21 & 19.77 \\
\hline Davidson Hata & $\mathbf{3 1 2 . 4 3}$ & $\mathbf{1 8 . 0 7}$ & $\mathbf{1 7 . 6 7}$ \\
\hline Egli & 955.48 & 31.60 & 30.91 \\
\hline Free Space & 3878.88 & 63.68 & 62.28 \\
\hline
\end{tabular}

\section{B. Comparison of Propagation Models with Terrain Data Resolution 30 Arc Second}

In this and following sections, terrain profile databases with various spatial resolutions and equivalent single knife edge diffraction have been used to calculate the diffraction factor and then evaluate their impact on the performance of the propagation models.

The results in Table 3 indicate that the Egli model can be considered the best fit to the measured data with low error when applying the diffraction factor on the propagation models. It can be clearly seen in Fig. 7 that the behavior of path loss was influenced by diffraction, compared with the path loss derived from measured data.

Thus, the propagation behaviour has been affected in most measurement locations when applying the terrain variation with 30 arc second $(1 \mathrm{~km})$ resolution. The impact of the propagation model is obvious after the third measurement point, where the first three points might be situated within the line of sight or the $1 \mathrm{~km}$ resolution results might have missed terrain features situated along the path which might cause destructive or constructive diffraction. Thus, using $1 \mathrm{~km}$ resolution might not give accurate results.

In Table 3, we can observe how the error statistics have been impacted by the diffraction factor and how the RMSE values are decreased in Egli and invreased in other propagation models. The results indicate that the Egli has the best results of the RMSE at $25.05 \mathrm{~dB}$. On the other hand, the Extended Hata model is seen to have less error compared with Davidson by about $5.7 \mathrm{~dB}$.

\section{Comparison of Propagation Models with Terrain Data Resolution 1 Arc Second}

Due to the nature of the terrain profile near the transmitter sites, which includes rough terrain and hills, the use of 1 arc second resolution (about $30 \mathrm{~m}$ ) will clearly affect the propagation predictions, as illustrated in Fig. 8. Here it may be seen clearly that there are large changes in the diffraction value at the distance of $48 \mathrm{~km}$ and that other locations such as the fifth location have less variation which might be placed in the line of sight of the transmitter. 


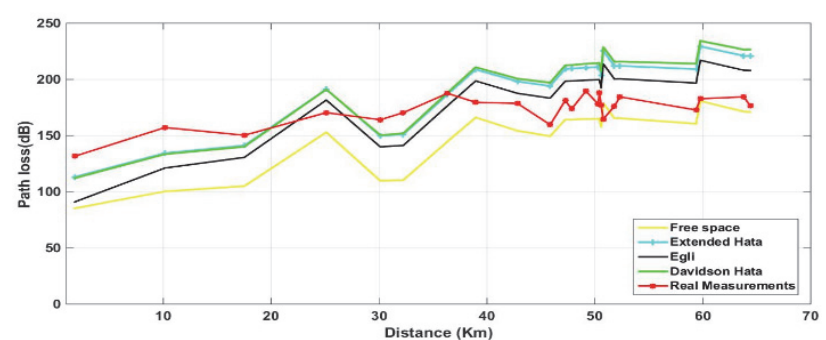

Fig. 7. Propagation models including diffraction by using terrain profile with resolution 30 Arc second.

TABle 3: Fitted Propagation Models InCluded TERRAIN 30 ARC SEC.

\begin{tabular}{|l|c|c|c|}
\hline Model & MSE & STD. Dev & RMSE \\
\hline Extended Hata & 945.90 & 31.44 & 30.75 \\
\hline Davidson Hata & 1138.66 & 34.50 & 33.74 \\
\hline Egli & $\mathbf{6 2 7 . 6 5}$ & $\mathbf{2 5 . 6 1}$ & $\mathbf{2 5 . 0 5}$ \\
\hline Free Space & 1432.08 & 38.69 & 37.84 \\
\hline
\end{tabular}

Therefore, it can be seen, when the resolution value of the terrain profile has increased, the error of the RMSE for all the models is decreased as observed by comparison of Tables 3 and 4.

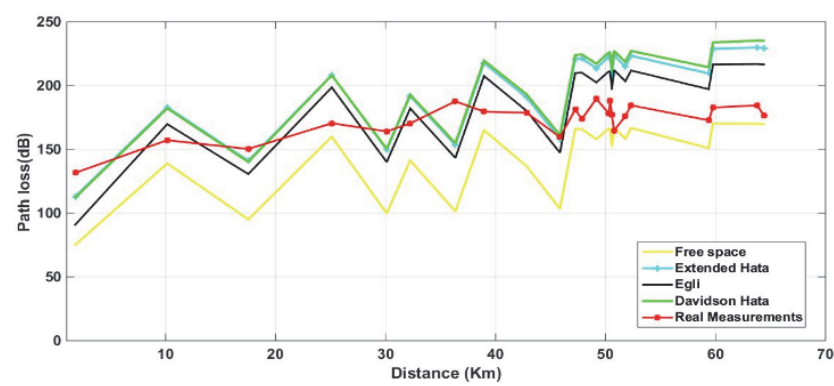

Fig. 8. Propagation models included diffraction by using terrain profile with resolution 1 Arc second.

TABle 4: Fitted Propagation Models InCluded TERRAIN 1 ARC SEC

\begin{tabular}{|l|c|c|c|}
\hline Model & MSE & STD. Dev & RMSE \\
\hline Extended Hata & 1273.87 & 36.49 & 35.69 \\
\hline Davidson Hata & 1480.35 & 39.34 & 38.47 \\
\hline Egli & $\mathbf{8 2 8 . 7 3 5}$ & $\mathbf{2 9 . 4 3}$ & $\mathbf{2 8 . 7 8}$ \\
\hline Free Space & 1229.02 & 35.84 & 35.05 \\
\hline
\end{tabular}

According to the advantage and disadvantage of both previous results in terms of accuracy and implementation time, we observed that, 30 arc second has short time and less accuracy, while 1 arc second has high accuracy and long implementation time. Therefore, the author proposed extra results which can improve the implementation time whilst maintaining reasonable accuracy by utilising a compromise between 30 and 1 arc second resolution.

\section{Comparison of Propagation Models with Terrain Data Resolution 3 Arc Second}

In the development work, we have selected and downloaded terrain data profile of 3 arc second, approximately $100 \mathrm{~m}$ resolution, which has less variation of the terrain data compared with 1 arc second, as shown in Fig. 9.

It can be observed in statistics of Table 5 that the use of 3 arc seconds, whilst giving a clear improvement in the implementation time, also impacts only slightly the accuracy of the results compared with the use of 1 arc second terrain data.

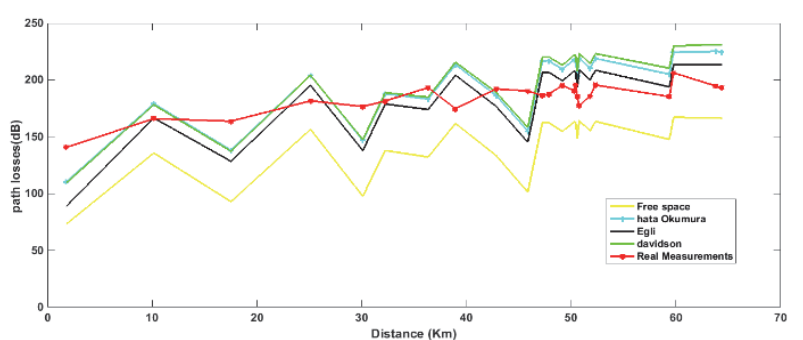

Fig. 9. Propagation models included diffraction by using terrain profile with resolution 3 Arc second.

Table 5: FitTed Propagation Models InCluded TERRAIN 3 ARC SEC

\begin{tabular}{|l|c|c|c|}
\hline Model & MSE & STD. Dev & RMSE \\
\hline Extended Hata & 1026.15 & 32.75 & 32.03 \\
\hline Davidson Hata & 1225.76 & 35.79 & 35.01 \\
\hline Egli & $\mathbf{6 4 4 . 4 5}$ & $\mathbf{2 5 . 9 5}$ & $\mathbf{2 5 . 3 8}$ \\
\hline Free Space & 1246.81 & 36.10 & 35.31 \\
\hline
\end{tabular}

Hence, according to these results, we observe clear sequences of the RMSE values for all models based on the terrain resolution, where the RMSE of Egli model was slightly increased $(25.05,25.38,28.78$ ) (30 Arc, 3 Arc, 1 Arc) respectively and has the lowest error compared with other propagation models in all terrain resolutions as illustrated in Table 3, 4 and 5.

\section{DESIGN OF FlEXIBLE SYSTEM FOR CREATING TVWS Database by USING DifFERENT Propagation MODELS}

Based on the previous results, which indicate that the Egli model is the best among the models that have been chosen for comparison with the real measurements, a flexible system has been built that performs many functions related to propagation modelling and calculation of signal strength in each pixel. Among these tasks, it is possible to determine any geographic area based on latitude and longitude between two concentric points. In addition, it can determine the size of each pixel which will affect the implementation time and propagation accuracy. Also, the system can perform three major operations at the same time to create a database for a selected geographic region that can be easily used when connected to the white space devices (WSD). In this work, for illustration, we considered only six transmitters, but more can be added using the "Add Transmitter Detail" button.

\section{A. Methodology for Calculation of Received Power}

The second methodology to be implemented after creating the pixel file is to calculate the receiver power in each pixel in the frequency range 470 to $790 \mathrm{MHz}$, by considering the selected transmitters. The processing time depends on the number of pixels, propagation model and also the terrain resolution level. The process will be conducted only once to create a database of the predicted TV signals in each pixel, as shown in Fig. 10, which can be used for the next stages.

\section{B. Methodology for Calculation of Available Channels}

The main goal of the system is to calculate available channels with high accuracy and then store all available channels of each pixel in the database, in a way which makes it easy to retrieve the data from WSDs. All of the 
transmitter information such as height, channels, transmitted power is stored previously in the database. The process takes into account all channels of the selected transmitters that might be received in a specific pixel, considering the weak signals as well.

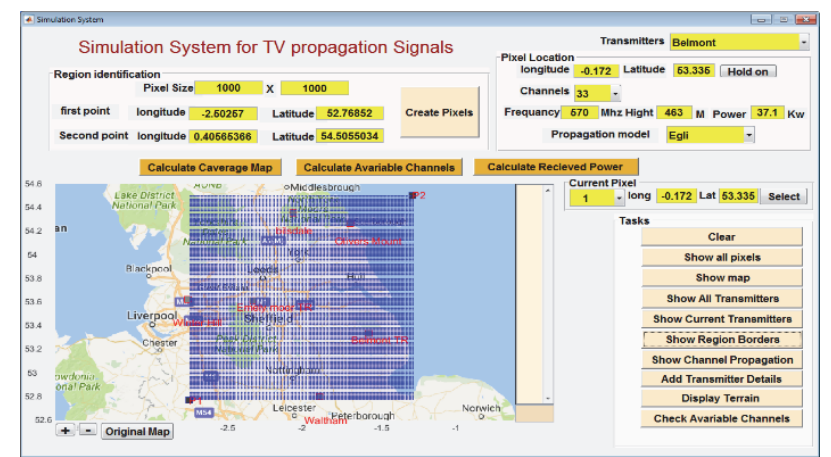

Fig. 10. Display all pixels in the selected resign in the simulation flexible system for creating TVWS database.

\section{Methodology for Calculation of Coverage Map}

This methodology must be calculated to translate the database that has been stored to show as a visual map of different levels of signal strength in the selected region for each transmitter and then stored in different files in the database as shown in Fig. 11.

\section{CONCLUSION}

In this paper, TV signal strengths are calculated using various propagation models and then compared with real measurements that have been conducted in various locations. Using a single knife edge model to calculate the diffraction factor with consideration of terrain profile data at different resolutions, we investigate and prove how the terrain data resolution impacts the accuracy and implementation time of the propagation models. We have improved and extended results that have been published in our conference paper in 2016 [10]. RMSE is the main criterion taken into account to assess the propagation models. The results summarized in Tables 3, 4 and 5 show that the Egli model still gives the best results when account is taken of the terrain profile data at a resolution of 3 arc second $(100 \mathrm{~m})$, providing lower values of RMSE compared with other propagation models, with shorter computation time and similar accuracy compared with 1 arc second. The other models all display relatively poor performance in terms of RMSE when terrain data at any resolution is considered. It may be seem that, for the terrain examples considered (which are all relatively smooth paths) the terrain resolution of 30 arc seconds is at least as good as the higher resolutions and it is therefore concluded that, in view of the much shorter computation time, this resolution will provide useful input to create a system for the cognitive radio decision process. However, we add the proviso that, should more irregular terrain be considered, the 3 arc second resolution may provide a better compromise between accuracy and computational time. In addition, the main benefits for designing the flexible system is to create a TVWS database for a specific area, by selecting the specific pixel size, adding appropriate transmitter information and choosing a suitable propagation model. In future work, we plan to implement additional propagation models and further investigate the effects of terrain resolution on channel selection accuracy.

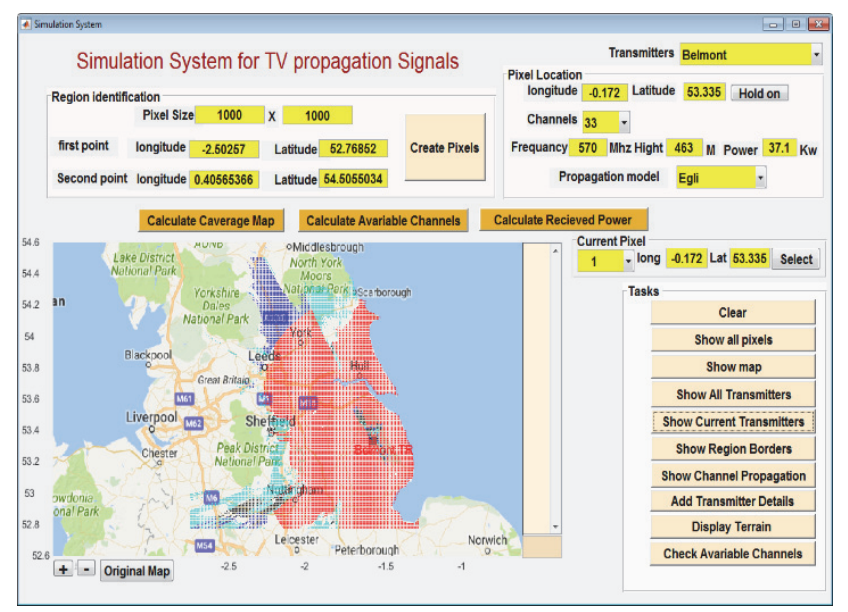

Fig. 11. Display the propagation signals of the channel 33 by using Egli model.

\section{ACKNOWLEDGMENT}

The author is grateful to his supervisor for his unlimited supporting to develop this study, by providing suitable solutions and suggestions.

\section{REFERENCES}

[1] D. Gurney, G. Buchwald, L. Ecklund, S. L. Kuffner, and J. Grosspietsch, "Geo-location database techniques for incumbent protection in the TV white space. In New Frontiers in Dynamic Spectrum Access Networks", DySPAN 2008. 3rd IEEE Symposium on (pp. 1-9), IEEE, October 2008.

[2] National Imagery and Mapping Agency, "Digital Terrain Elevation Data“, 30 May, 1990,

www.globalsecurity.org/ intell/systems/dted.htm.

[3] M. Nekovee, "A survey of cognitive radio access to TV white spaces", In International Conference on Ultra Modern Telecommunications \& Workshops (pp. 1-8, IEEE, October 2009.

[4] M. F. Iskander and Z. Yun. "Propagation prediction models for wireless communication systems. Microwave Theory and Techniques “, IEEE Transactions on, 50(3), pp.662-673, March 2002.

[5] P. Pardeep, P. Kumar and B. S. Rana, "Performance evaluation of different path loss models for broadcasting applications", American Journal of Engineering Research (AJER), 3(4), pp.335-342, 2014.

[6] K. Stylianos, P. I. Lazaridis, Z. D. Zaharis, A. Bizopoulos, S. Zettas, and J. Cosmas, "Comparison of Longley-Rice, ITU-R P. 1546 and Hata-Davidson propagation models for DVB-T coverage prediction", In BMSB, pp. 1-4, 2014.

[7] M. Hope, A. B. Bagula, M. Zennaro, and G. Lusilao-Zodi, "On the impact of propagation models on TV white spaces measurements in Africa“, International Conference on. IEEE, pp.148-154, May 2015.

[8] P. Prajesh, and R. K. Singh, "Investigation of outdoor path loss models for wireless communication in Bhuj", International Journal of Electronics and Communication Engineering \& Technology (IJECET), Volume 3, Issue 2, pp. 171-178, September 2012.

[9] J. You-Cheol, "Diffraction analysis and tactical applications of signal propagation over rough Terrain", Air Force INST of Tech WrightPatterson AFB OH School of Engineering, no. 97J-01, Dec 1997.

[10] A. M. Fanan, N. Riley, M. Mehdawi and M. Ammar, "Comparison of propagation models with real measurement around Hull, UK," 2016 24th Telecommunications Forum (TELFOR), Belgrade, 2016, pp. 1-4. 